\title{
Arteria Ulnar Superficial, Bilateral y Antebraquial. Similitudes y Diferencias
}

\author{
Superficial Ulnar Artery, Bilateral and Antebrachial. Similarities and Differences
}

Homero Felipe Bianchi ${ }^{1,2}$; Claudia Andrea $\operatorname{Vargas}^{3,4}$ \& Nicolás Ernesto Ottone ${ }^{5,6}$

BIANCHI, H. F.; VARGAS, C. A. \& OTTONE, N. E. Arteria ulnar superficial, bilateral y antebraquial. Similitudes y diferencias. Int. J. Morphol., 36(3):997-1001, 2018.

RESUMEN: Describimos un hallazgo infrecuente de arteria ulnar superficial en ambos antebrazos de un cadáver, con origen en la arteria braquial, a nivel de la fosa cubital. Las arterias presentan un trayecto que dividimos en cuatro segmentos, a causa de sus flexuosidades. Ambos antebrazos presentan ausencia del músculo palmaris longus. Se resumen las principales teorías sobre su desarrollo y las complicaciones que pueden ser ocasionadas por su presencia y el eventual beneficio de la misma. Hasta el momento, no hemos encontrado en la literatura, una disposición morfológica de arterias ulnares superficiales como las aquí descritas.

PALABRAS CLAVE: Arteria ulnar superficial; Miembro superior; Variaciones anatómicas.

\section{INTRODUCCIÓN}

Durante una disección de rutina, observamos una arteria ulnar superficial (AUS) bilateral, en un espécimen de sexo masculino, de 64 años, fresco, con el sistema arterial inyectado con látex natural coloreado con pigmento rojo, conservado a posteriori en formalina al $10 \%$.

El origen de la AUS ha sido reportado en la arteria axilar (AA) (Dubreuil Chambardel, 1926; Singer, 1933; Weathersby, 1956; Fadel \& Amonoo-Kuofi, 1996; Jacquemin et al., 2001; Natsis et al., 2006; Mannan et al., 2008; Yadav et al., 2013); en la arteria braquial (AB) en el brazo (Hazlett, 1949; Weathersby; Blagojka, 1982; del Sol et al., 2000; Rodríguez-Niedenführ et al., 2001; Natsis et al.; Dartnell et al., 2007; Senanayake et al., 2007; Troupis et al., 2011; Casal et al., 2012; Solan, 2013; Salunke et al., 2014; Abhinita et al., 2016) o en la fosa cubital (Hazlet; Weathersby; Blagojka; Panicker et al., 2003; Shetty et al., 2013; Yadav et al.; Kusztal et al., 2014; Kumka \& Purkiss, 2015; Quadros et al., 2015; Sirasanagandla et al., 2016). En nuestro caso, los hallazgos remiten a este último origen y al trayecto en el antebrazo de ambas arterias.

\section{REPORTE DE CASOS}

Antebrazo izquierdo. La AUS (Fig. 1) nace de la AB, previo a su división en arteria radial (AR) y tronco de las arterias interóseas (TAIO), $6 \mathrm{~cm}$ distal a la línea biepicondílea. Por los cambios en su trayectoria podemos considerar cuatro segmentos: el primer segmento, desde su origen, describe primero una curva a medial de $1,5 \mathrm{~cm}$ de longitud, seguida de un trayecto recto de $3 \mathrm{~cm}$, continuado por una segunda curva de igual dirección que la primera, de $1 \mathrm{~cm}$ de longitud, ambas en forma de $\mathrm{S}$ invertida. La distancia al epicóndilo medial (EPCM) es de $6 \mathrm{~cm}$ la primera y de 10 $\mathrm{cm}$ la segunda. El segundo segmento, casi recto (con una ligera curva en su parte central) de $16 \mathrm{~cm}$ de largo, se extiende hasta una tercera curva, $5 \mathrm{~cm}$ proximal al retinaculo flexor (RF), seguida de un tercer segmento transversal de dirección hacia medial, casi recto, de $2 \mathrm{~cm}$ de longitud. Termina en una cuarta curva, seguida de un cuarto y último segmento recto de $3 \mathrm{~cm}$ de longitud, continuado por el arco palmar superficial (APS) (Fig. 2A). El primer segmento se encuentra superficial a los músculos insertos en el EPCM: pronador redondo (PR), flexor superficial de los dedos (FSD) y flexor radial del carpo (FRC). Hay ausencia del músculo

\footnotetext{
${ }^{1}$ Cirujano de Mano, Profesor Consulto, Departamento de Anatomía, Facultad de Medicina, Universidad de Buenos Aires, Buenos Aires, Argentina. ${ }^{2}$ Departamento de Anatomía, Fundación Barceló, Instituto Universitario de Ciencias de la Salud, Buenos Aires, Argentina.

${ }^{3}$ Departamento de Educación Física, Deportes y Recreación, Facultad de Educación, Universidad de La Frontera, Temuco, Chile.

${ }^{4}$ Programa de Doctorado en Ciencias Morfológicas, Facultad de Medicina, Universidad de La Frontera, Temuco Chile.

${ }^{5}$ Laboratorio de Plastinación y Técnicas Anatómicas, Centro de Investigación en Ciencias Odontológicas (CICO), Facultad de Odontología, Universidad de La Frontera, Temuco, Chile.

${ }^{6}$ Centro de Excelencia en Estudios Morfológicos y Quirúrgicos (CEMyQ), Facultad de Medicina, Universidad de La Frontera, Temuco, Chile.
} 

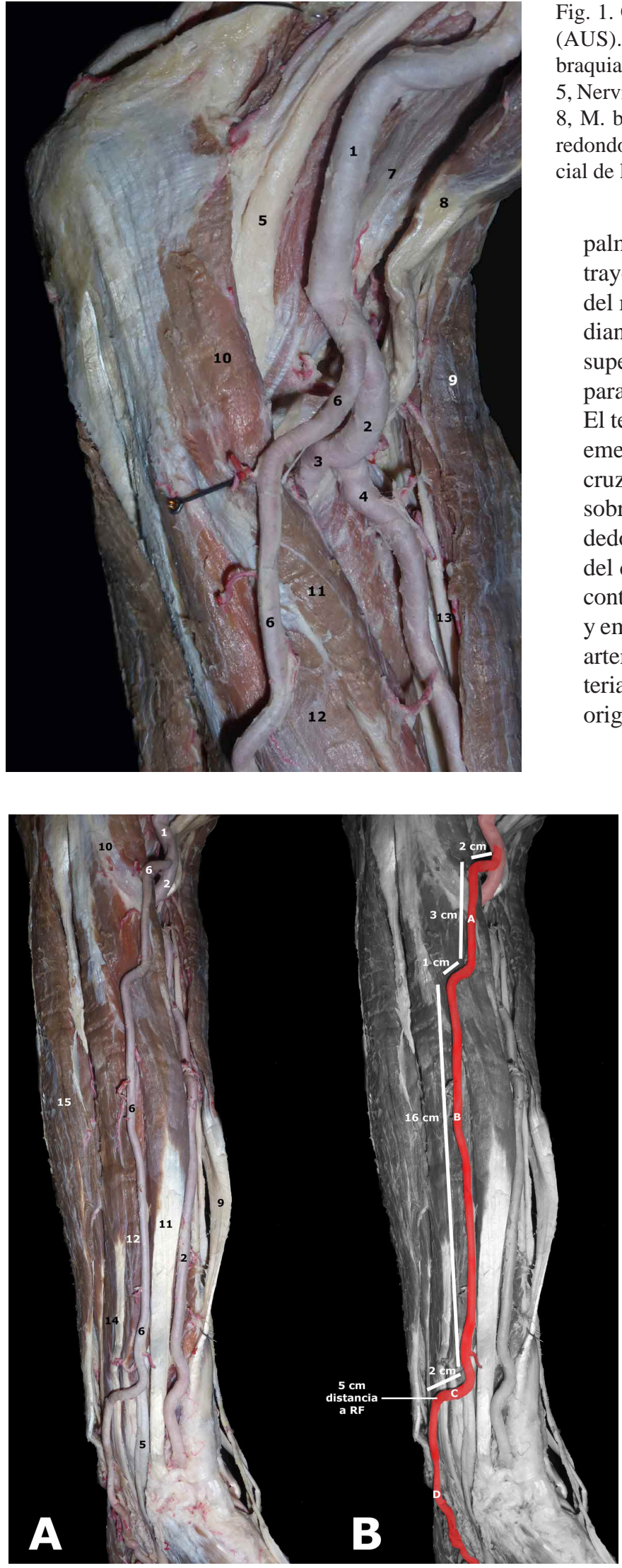

Fig. 2. A. Segmentos de la arteria ulnar superficial (AUS) en el antebrazo izquierdo con sus características y relaciones con los tendones y músculos. 1, Arteria braquial; 4, Arteria radial; 5, Nervio mediano; 6, Arteria ulnar superficial; 9, M. braquioradial; 10, M. pronador redondo; 11, M. flexor radial del carpo; 12, M. flexor superficial de los dedos; 14, M. flexor profundo de los dedos; 15, M. flexor ulnar del carpo. B. Segmentos de la AUS y sus mediciones en el antebrazo izquierdo. A, B, C, D: primero, segundo, tercero and cuarto segmento de la AUS con sus medidas en centímetros. RF, retináculo flexor. 


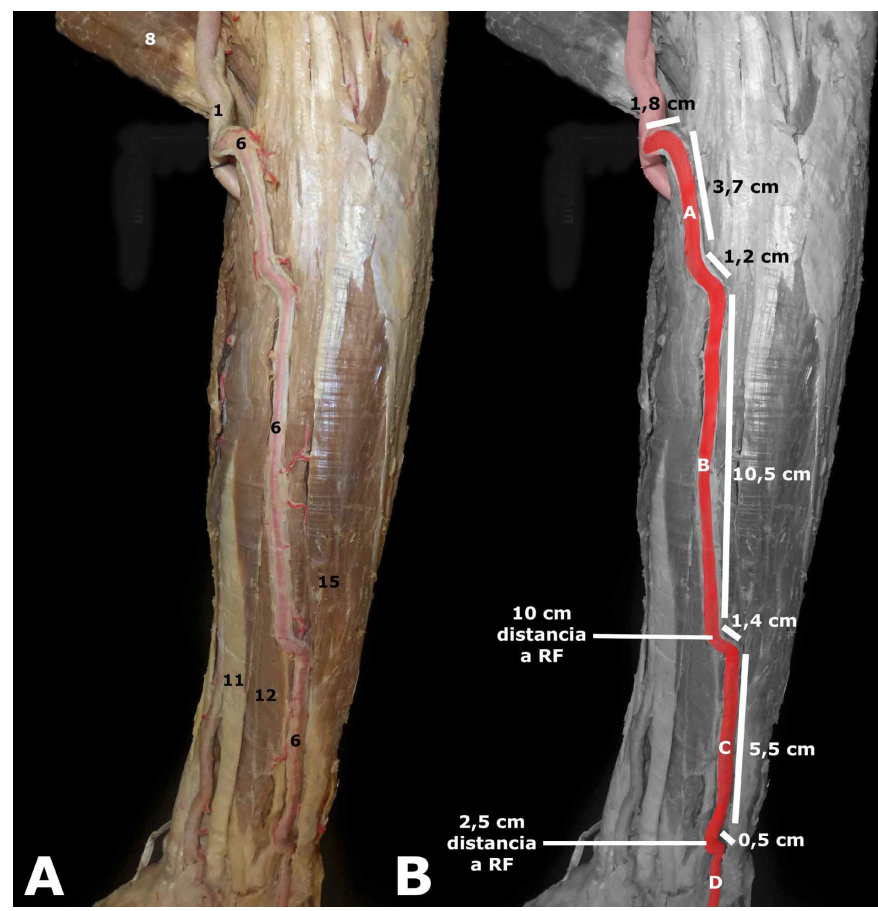

Fig. 3. A. Segmentos de la AUS en el antebrazo derecho con sus características y relaciones con los tendones y músculos. 1, Arteria braquial; 8, M. bíceps braquial; 11, M. flexor radial del carpo; 12, M. flexor superficial de los dedos; 15, M. flexor ulnar del carpo. B. Segmentos de la AUS y sus mediciones en el antebrazo derecho. A, B, C, D: primero, segundo, tercero and cuarto segmento de la AUS con sus medidas en centímetros. RF, retináculo flexor.

cuyo tendón sigue, continuándose el tercer segmento, que presenta una longitud de $5,5 \mathrm{~cm}$, hasta una curva de $0,5 \mathrm{~cm}$ hacia anterior, $2,5 \mathrm{~cm}$ proximal al RF, después de la cual podríamos admitir un cuarto segmento de longitud similar al lado izquierdo, que llegado a la mano forma el APS (Fig. 3B). La disposición del TAIO y sus ramas y de la AR y sus ramas es igual al lado izquierdo.

En ambos lados se comprobó la no existencia de una arteria ulnar de trayecto habitual.

\section{DISCUSIÓN}

La presencia bilateral de la AUS ha sido mencionada en la literatura del siguiente modo: originándose ambas AUS de la AB en el brazo (del Sol et al.; Dartnell et al.); naciendo ambas AUS de la arteria axilar (AA) (Fadel \& Amonoo-Kuofi; Jacquemin et al.); o con diferente origen, una AUS originándose de la AA, y la contralateral naciendo de la $\mathrm{AB}$ en la fosa cubital (Yadav et al.). Solan describe las AUS naciendo de la $\mathrm{AB}$, una en el brazo y la otra en la fosa cubital.
En nuestro trabajo, describimos un nuevo caso de AUS bilateral de igual origen, naciendo ambas de la $\mathrm{AB}$ a nivel de la fosa cubital, hecho aún no mencionado en la literatura. En nuestros hallazgos no encontramos una arteria ulnar de origen y trayecto habitual, lo cual ha sido señalado en forma infrecuente (Mannan et al.; Solan).

En nuestros especímenes, el origen de las AUS en la fosa cubital fue del lado medial de la AB, a una distancia de $6 \mathrm{~cm}$ de la línea biepicondilea. En ambos casos, la AR emite la arteria recurrente radial y el TAIO, que se divide en AIOA y AIOP, lo que concuerda con lo hallado por otros autores (Dubreuil Chambardel; Hazlett; Weathersby; del Sol et al.; Jacquemin et al.; Natsis et al.; Dartnell et al.; Senanayake et al.; Ashim et al.; Kumka \& Purkiss; Quadros et al.; Abhinita et al.; Sirininvasa et al.). Las muestras evidenciaron a la arteria recurrente ulnar posterior en ambas preparaciones, pero no se encontró la arteria recurrente ulnar anterior.

Ambas AUS muestran tortuosidades, descritas previamente (Mannan et al.; Solan; Rodrigues et al., 2016) pero con morfología diferente a nuestros hallazgos, sin establecer la causa de la deformación, siendo interpretadas como deficiencias del tejido conectivo. Las tortuosidades del primer segmento en ambos casos son similares en morfología y extensión, con ligera oblicuidad la del antebrazo derecho. En los restantes segmentos, las arterias mostraron flexuosidades, siendo notoria la del lado izquierdo, proximal al RF, de la que no encontramos mención en la literatura, posición que la deja muy expuesta a los abordajes volares de la muñeca y del tercio inferior del antebrazo. Hay una referencia dudosa en Quain (1884) ya que no se acompaña de representación gráfica. A la derecha, la presencia de la última curva es la causa de que la AUS izquierda tenga 4 segmentos al subdividir al tercer segmento en dos. Si la comparamos con el lado opuesto, su menor desarrollo la hace menos expuesta al trauma quirúrgico.

La ubicación de los segundos segmentos en ambos antebrazos fue superficial a los músculos antebraquiales, siendo el trayecto recto y central hasta la mencionada flexura en el izquierdo, y oblicuo en el derecho (Dartnell et al.; Mannan et al.; Casal et al.; Shetty et al.).

La ubicación superficial de la AUS se relaciona con la ausencia del músculo PL, no encontrado en ambos especímenes, hecho mencionado por varios autores (Fadel \& Amonoo-Kuofi; Jacquemin et al.; Mannan 
et al.; Yadav et al.; Abhijee et al.; Abhinita et al.; Srininvasa et al.). Yadev considera su ausencia como un signo de advertencia de la presencia de una AUS. Por el contrario, cuando el PL está presente, la AUS se ubica por debajo de los músculos antebraquiales (Quain; Sieg et al., 2006; Weathersby; Dartnell et al.; Solan; Srininvasa et al.).

Por otro lado, según la definición establecida por Ottone et al. (2006) al identificar variaciones anatómicas de las arterias hepáticas, la AUS podría definirse como una arteria ulnar aberrante reemplazante superficial, identificando como "aberrantes" a las arterias que tienen un origen distinto al considerado clásico o normal, y "reemplazante", debido a que reemplaza al vaso de disposición normal, teniendo además en este caso un trayecto anómalo, al ser superficial.

Diversas teorías tratan de explicar las variedades arteriales, destacándose la teoría de los brotes vasculares de Singer a partir de un eje axial, comunicados por una red capilar, a la que se suman otros autores (Fadel \& AmonooKuofi; Jacquemin et al.; Panicker et al.; Quadros et al.), y la teoría de Rodríguez-Niedenführ et al. sostenida por otros autores como Dartnell et al., Senanayake et al., Casal et al. y Kumka \& Purkiss, quienes postulan la presencia de una red primitiva vascular que se ramifica y anastomosa por canales capilares siguiendo el desarrollo del miembro superior. En ambas teorías existe un desarrollo, involución y desaparición de canales capilares; cuando aquellos que deberían desaparecer no lo hacen y en cambio se desarrollan, serían el origen de las variedades arteriales.

Si bien la incidencia reportada es baja $(0,7-9,4 \%)$ (Natsis et al.; Senanayake et al.; Dartnell et al.; Ramani et al., 2014; Kumka \& Purkiss; Quadros et al.), los problemas derivados de su presencia han sido reportados por la mayoría de los autores. De las referencias surgen dos como los más frecuentes: la confusión con una vena y la administración intravenosa de medicamentos, con riesgo de isquemia, base de la comunicación de Hazlett con la isquemia resultante (Fadel \& Amonoo-Kuofi; del Sol et al.; Jacquemin et al.; Natsis et al.; Senanayake et al.; Abhijee et al.; Kumka \& Purkiss; Abhinita et al.) y la inclusión de la arteria en un colgajo cutáneo vascularizado radial que dañe o incluya a la AUS, dejando al miembro superior sin circulación distal (Fadel \& Amonoo-Kuofi; Natsis et al.; Dartnell et al.; Mannan et al.; Abhijee et al.; Abhinita et al.) ya que como se mencionó, es excepcional la presencia de una arteria ulnar normal. Mannan et al. considera que el uso del torniquete en estas circunstancias puede inducir a error. Otras lesiones son las derivadas de la introducción de catéteres en procedimientos hemodinámicos, en especial en donde existen tortuosidades
(Jacquemin et al.; Dartnell et al.; Abhinita et al.), desarrollo de trombosis y aneurismas por la presencia de inflexiones (Fadel \& Amonoo-Kuofi), dificultades en el diagnóstico de imágenes, en la toma de arteria radial para el bypass coronario (Kitshiri et al.; Dartnell et al.; Abhinita et al.), daños en abordajes quirúrgicos y trauma, en particular en una presentación como la descrita en el antebrazo izquierdo, como señalamos previamente (Dartnell et al.; Senanayake et al.; Casal et al.). Por el contrario, su presencia se considera ventajosa cuando se la utiliza en el tallado de colgajos cutáneos vascularizados antebraquiales preservando a la arteria radial (Fadel \& Amonoo-Kuofi; Jacquemin et al.; Natsis et al.; Bell et al., 2011; Ramani et al.; Rodrigues et al.). Para Kusztal et al. la presencia de esta variación no es contraindicación de la fistula arteriovenosa para la hemodiálisis.

Es posible determinar su presencia en el examen, en especial en pacientes delgados, por la onda pulsátil que nos pone en aviso de su presencia; en caso de duda, se aconseja el uso de eco doppler (Dartnell et al.; Bell et al.; Abhijee et al.; Kumka \& Purkiss) para mejorar la planificación de la cirugía a efectuar.

\section{CONCLUSIONES}

Es necesario tener presente esta variedad de disposición arterial, ante la posibilidad de graves complicaciones derivadas de su trauma o confusión con venas seguida de administración de medicamentos, descriptas ampliamente en todas las referencias consultadas. Su ventaja es su uso en el tallado de colgajos antebraquiales vascularizados. Aunque no es de rutina, debería tenerse en consideración el uso del eco doppler para un mejor planeamiento preoperatorio.

BIANCHI, H. F.; VARGAS, C. A. \& OTTONE, N. E. Superficial ulnar artery, bilateral and antebrachial. Similarities and differences. Int. J. Morphol., 36(3):997-1001, 2018.

SUMMARY: We describe an infrequent finding of a superficial ulnar artery in both cadaver forearms, which originates in the brachial artery at cubital fossa level. The arteries have a path that we divided into four segments, because of their flexuosities. Both forearms showed an absence of the palmaris longus muscle. This summarizes the main theories about its development and the complications and eventual benefits that can be caused by its presence. So far, we have not found in the literature, a morphological disposition of superficial ulnar arteries such as those described here.

KEY WORDS: Superficial ulnar artery; Upper limb; Anatomical variations. 


\section{REFERENCIAS BIBLIOGRÁFICAS}

Abhinita, P.; Kumar, N.; Mohandas Rao, K. G.; Satheesha Nayak, B.; Ravindra, S. \& Ashwini Aithal, P. High origin of superficial ulnar artery associated with absence of palmaris longus muscle- a rare concurrent anatomical variation. Online J. Health Allied Sci., 15(1):1-3, 2016.

Bell, R. A.; Schneider, D. S. \& Wax, M. K. Superficial ulnar artery: a contraindication to radial forearm free tissue transfer. Laryngoscope, 121(5):933-6, 2011.

Blagojka, K. J. Angiography of the upper extremity. London, Martinus Nijhoff Publisher, 1982.

Casal, D.; Pais, D.; Toscano, T.; Bilhim, T.; Rodrigues, L.; Figueiredo, I.; Aradio, S.; Angélica-Almeida, M. \& Goyri-O'Neill, J. A rare variant of the ulnar artery with important clinical implications: a case report. $B$. M. C. Res. Notes., 5:660, 2012.

Dartnell, J.; Sekaran, P. \& Ellis, H. The superficial ulnar artery: incidence and calibre in 95 cadaveric specimens. Clin. Anat., 20(8):929-32, 2007.

del Sol, M.; Olave, E.; Hettich, M. E. \& Bustos, K. Superficial ulnar artery. Anatomoclinical importancy. Rev. Chil. Anat., 18(1):85-8, 2000.

Dubreuil Chambardel, L. Variations des Artères du Member Supérieur. Paris, Masson, 1926.

Fadel, R. A. \& Amonoo-Kuofi, H. S. The superficial ulnar artery: Development and surgical significance. Clin. Anat., 9(2):128-32, 1996.

Hazlett, J. W. The superficial ulnar artery with reference to accidental intraarterial injection. Can. Med. Assoc. J., 61(3):289-93, 1949.

Jacquemin, G.; Lemaire, V.; Medot, M. \& Fissette, J. Bilateral case of superficial ulnar artery originating from axillary artery. Surg. Radiol. Anat., 23(2):139-43, 2001.

Kumka, M. \& Purkiss, S. A rare case of unilateral variations of forearm arteries: anatomy, embryology and clinical implications. J. Can. Chiropr. Assoc., 59(3):253-60, 2015.

Kusztal, M.; Weyde, W.; Letachowicz, K.; Goebiowski, T. \& Letachowicz, $\mathrm{W}$. Anatomical vascular variations and practical implications for access creation on the upper limb. J. Vasc. Access, 15 Suppl. 7:S70-5, 2014.

Mannan, A.; Sarikcioglu, L.; Ghani, S. \& Hunter, A. Superficial ulnar artery terminating in a normal ulnar artery. Clin. Anat., 18(8):602-5, 2008.

Natsis, K.; Papadopoulou, A. L.; Paraskevas, G.; Totlis, T. \& Tsikaras, P. High origin of a superficial ulnar artery arising from the axillary artery: anatomy, embryology, clinical significance and a review of the literature. Folia Morphol. (Warsz), 65(4):400-5, 2006.

Ottone, N. E.; Arrotea Molina, A.; Domínguez, M. L.; Lo Tartaro, M.; Garcia De Quiros, N.; Medan, C. Blasi, E. \& Bertone, V. H. Aberrants hepatic arteries. Research over 64 disections. Int. J. Morphol., 24(4):581-585, 2006.

Panicker, J. B.; Thilakan, A. \& Chandi, G. Ulnar artery: A case report of unusual origin and course. J. Anat. Soc. India, 52(2):177-9, 2003.

Quadros, L. S.; Bhat, N. \& D'Souza, A. S. Superficial ulnar artery: A case report of its unusual course. Malays. J. Med. Sci., 22(3):65-7, 2015.

Quain, R. The Anatomy of the Arteries of the Human Body. London, Taylor and Walton, 1884.

Ramani, C. V.; Kundagulwar, G. K.; Prabha, Y. S. \& Dushyanth, J. Anomalous superficial ulnar artery based flap. Indian J. Plast. Surg., 47(1):124-6, 2014.

Rodrigues, V.; Rao, M. K.; Nayak, S. \& Kumar, N. Looped and Tortuous Ulnar Artery - An Erratic Unilateral Vascular Presentation in the Proximal Forearm. J. Clin. Diagn. Res., 10(6):AD03-4, 2016.

Rodríguez-Niedenführ, M.; Burton, G. J.; Deu, J. \& Sañudo, J. R. Development of the arterial pattern in the upper limb of staged human embryos: normal development and anatomic variations. J. Anat., 199(Pt. 4):407-17, 2001.

Salunke, A. A.; Nambi, G. I.; Dhanwate, A. D. \& Siriwardana, H. A. Superficial ulnar artery: Clinical recommendations to avoid iatrogenic complications due to variation in arterial system. Niger. Med. J.,
55(3):276-7, 2014

Senanayake, K. J.; Salgado, S.; Rathnayake, M. J.; Fernando, R. \& Somarathne, K. A rare variant of the superficial ulnar artery, and its clinical implications: a case report. J. Med. Case Rep., 1:128, 2007.

Shetty, S. D.; Nayak, B. S.; Ashwini, L. \& Sirasanagandla, S. R. Variant origin and course of ulnar artery - a case report. Int. J. Anat. Var., 6:456, 2013.

Sieg, P.; Jacobsen, H. C.; Hakim, S. G. \& Hermes, D. Superficial ulnar artery: curse or blessing in harvesting fasciocutaneous forearm flaps. Head Neck, 28(5):447-52, 2006.

Singer, E. Embryological pattern persisting in the arteries of the arm. Anat. Rec., 55(4):403-9, 1933.

Sirasanagandla, S. R.; Pamidi, N.; Nayak, S. B.; Jetti, R. \& Thangarajan, R. Superficial ulnar artery associated with anomalous origin of the common interosseous and ulnar recurrent arteries. J. Clin. Diagn. Res., 10(5):AD01-2, 2016.

Solan, S. Accessory superficial ulnar artery: a case report. J. Clin. Diagn. Res., 7(12):2943-4, 2013.

Troupis, T. G.; Tsakotos, G.; Michalinos, A.; Piagkou, M.; Johnson, E. O.; Xanthos, T. \& Skandalakis, P. Uncommon course of the ulnar artery. Am. Surg., 77(12):1717-9, 2011.

Weathersby, H. T. Unusual variation of the ulnar artery. Anat. Rec., 124(2):245-8, 1956.

Yadav, P. S.; Ahmad, Q. G.; Shankhdhar, V. K. \& Nambi, G. I. Absence of the palmaris longus is a warning sign for avoiding the superficial ulnar artery 'trap'. Indian J. Plast. Surg., 46(1):149-50, 2013

Dirección para correspondencia:

Dr. Nicolás E. Ottone

Laboratorio de Plastinación y Técnicas Anatómicas

Centro de Investigación en Ciencias Odontológicas (CICO)

Facultad de Odontología

Universidad de La Frontera

Temuco

CHILE

Email: nicolas.ottone@ufrontera.cl

Recibido : 05-04-2018

Aceptado: 19-06-2018 